\title{
Dynamical quantum depletion in polariton condensates
}

\author{
Selma Koghee and Michiel Wouters \\ Theory of Quantum and Complex Systems, Universiteit Antwerpen, Universiteitsplein 1, B-2610 Antwerpen, Belgium
}

(Received 2 July 2015; revised manuscript received 14 October 2015; published 24 November 2015)

\begin{abstract}
We present a theoretical study of the quantum depletion of microcavity polaritons that are excited with a resonant laser pulse. The dynamics of the quantum fluctuations are interpreted in the context of quantum quenches in general and in terms of the dynamical Casimir effect in particular. We compute the time evolution of the first- and second-order correlation functions of the polariton condensate. Our theoretical modeling is based on the truncated Wigner approximation for interacting Bose gases. For homogeneous systems, analytical results are obtained in the linearized Bogoliubov approximation. Inhomogeneous systems are studied numerically by Monte Carlo simulations.
\end{abstract}

DOI: 10.1103/PhysRevB.92.195309

PACS number(s): 71.36.+c, 03.75.Kk, 42.25.Kb, 42.50.Lc

\section{INTRODUCTION}

Interaction quenches in quantum many-body systems have become an active research field [1,2], mainly thanks to the great degree of controllability of ultracold atoms with Feshbach resonances and optical lattices [3]. Recently, a complementary platform for quantum many-body physics has been developed, namely, exciton-polariton quantum fluids [4]. The distinctive characteristic of these systems is that the polaritons are a superposition of light and matter excitations. A first advantage of the light component is that it allows for a straightforward diagnostic of the fluid by means of standard quantum optical techniques. A second advantage is that polaritons can be created by an external laser field. It is this feature of polariton condensates that is of particular interest in the context of quantum quenches, since it allows one to implement a sudden change in the many-body system.

The situation that we consider here is an instantaneous injection of polaritons in a coherent state [5]. Since this state is not the ground state of a weakly interacting (lossless) many-body system, a nontrivial time evolution will result due to interactions, in addition to the decay because of the losses. In our previous work, we have shown that a dynamical Casimir effect [6-8] takes place in terms of the Bogoliubov excitations on top of the coherently created polariton state. Indeed, the sudden creation of a condensate quenches the vacuum from the trivial one to the Bogoliubov vacuum, resulting in an excitation of the system. Part of the motivation for the study of the dynamical Casimir effect stems from connections with the Hawking-Unruh effect, whose sonic version [10] is getting within the reach of experiments with polaritons [9] and ultracold atoms [11].

The analogy with an interaction quench in cold atom systems is direct, since our proposal is equivalent to a sudden increase of the interaction strength, from zero to a finite value. Such experiments have been performed with ultracold atoms by Hung et al. [12], who suddenly decreased the interaction strength in an atomic Bose-Einstein condensate. The resulting density oscillations were related to Sakharov oscillations in the early universe [13].

An even closer connection can be made with the splitting quench by Langen et al. in one-dimensional atomic condensates [14]. When a condensate is rapidly split into two parts, there is initially perfect phase coherence between them.
However, at later times, the two parts start to develop a different phase. This dephasing due to interactions is entirely analogous to the one in our dynamical Casimir proposal, showing a light-cone-like emergence of thermal correlations.

An important difference between polaritonic and atomic condensates concerns the ratio of the lifetime to the characteristic time scale of the dynamics. Whereas for ultracold atoms, this ratio is very large, in polariton systems losses are more important. Their theoretical modeling should therefore be carried out in an open system setting. This raises the interesting issue of the competition between losses and thermalization dynamics.

We will treat the open system quantum dynamics within the truncated Wigner approximation, which is a popular tool in both the study of conservative cold atoms $[15,16]$ and for lossy polariton systems [4]. When the condensate depletion is small, the equations of motion can be linearized in the fluctuations, which is equivalent to the Bogoliubov approximation.

In Sec. II, we use this approximation to obtain analytical results for the first- and second-order coherence functions in the homogeneous system. For the inhomogeneous case, we perform in Sec. IV Monte Carlo simulations of the stochastic equations of motion. We show that for a large smooth pumping spot, a local density approximation satisfactorily reproduces the first-order coherence function. Conclusions are drawn in Sec. V.

\section{THE MODEL}

We consider a driven dissipative bosonic system, whose dynamics is governed by a master equation of the Lindblad type:

$$
\frac{d}{d t} \rho=-\frac{i}{\hbar}[H, \rho]+\mathcal{D}(\rho) .
$$

Here, the Hamiltonian $H=H_{P}+H_{L}$ contains the free Bose gas dynamics of the polaritons

$$
H_{P}=\int d x \psi^{\dagger}(x)\left[\frac{-\hbar^{2}}{2 m} \nabla^{2}+\frac{g}{2} \psi^{\dagger}(x) \psi(x)\right] \psi(x),
$$

with $m$ being the lower polariton effective mass and $g$ the interaction strength. $\psi(x)$ is the annihilation operator for a polariton at position $x$. The Hamiltonian also includes the 
external laser driving,

$$
H_{L}=\int d x\left[F_{L}(x, t) \psi^{\dagger}(x)+F_{L}^{*}(x, t) \psi(x)\right],
$$

where $F_{L}$ is the laser amplitude.

The polariton losses, which depend on the linewidth $\gamma$, are described by the dissipator $\mathcal{D}(\rho)$, that we take to be of Lindblad form:

$$
\begin{aligned}
\mathcal{D}(\rho)= & \int d x \frac{\gamma}{2 \hbar}\left[2 \psi(x) \rho \psi^{\dagger}(x)\right. \\
& \left.-\psi^{\dagger}(x) \psi(x) \rho-\rho \psi^{\dagger}(x) \psi(x)\right] .
\end{aligned}
$$

When a microcavity is excited sufficiently close to the lower polariton branch and all the relevant energy scales (linewidth, interaction energy) are much smaller than the Rabi splitting, it is well justified to restrict the dynamics to the lower polariton branch.

For the quantum quench that we consider, we take the driving laser to be an ultrashort pulse. When the pulse duration $\delta t$ is much shorter than all the other time scales of the dynamics, shortly after the pulse, the polariton field is in a coherent state with amplitude $\psi_{0} \equiv\langle\psi(x, t=0)\rangle=$ $\int_{-\delta t}^{0} F_{L}(x, t) d t$. The external laser drive then only sets the initial condition and does not affect the polariton dynamics, which is governed by the free Bose gas dynamics and the losses only.

We will solve the master equation (1) within the truncated Wigner approximation (TWA) [16,17], a method that is widely used for the simulation of weakly interacting one-dimensional atomic condensates. The addition of losses makes the TWA an even better approximation to the exact dynamics.

The resulting stochastic equations of motion read [5]

$$
\begin{aligned}
i \hbar d \phi(x, t)= & {\left[-\frac{\hbar^{2} \nabla^{2}}{2 m}-i \frac{\gamma}{2}+g|\phi(x, t)|^{2}\right] \phi(x, t) d t } \\
& +\sqrt{\frac{\hbar \gamma}{4 \Delta V}} d W(x, t),
\end{aligned}
$$

where $\Delta V$ is the volume of a single cell of the discretized grid. The complex Gaussian noise $d W(x, t)$ satisfies $\left\langle d W^{*}(x, t) d W\left(x^{\prime}, t^{\prime}\right)\right\rangle=2 d t \delta_{t, t^{\prime}} \delta_{x, x^{\prime}} /(\Delta V)$, and all other combinations average to zero. Since the expectation values of the stochastic fields are equal to the symmetrized averages of the quantum fields, the TWA can be used to study the quantum fields. The relation between the stochastic and quantum fields also allows us to calculate the initial expectation values of the former: $\left\langle\phi^{*}(x, t=0) \phi(x, t=0)\right\rangle=n(x, t=0)+1 / 2$, where $n(x, t=0)$ is the initial density distribution.

\section{HOMOGENEOUS SYSTEM}

First we will consider a homogeneous system, where we assume a homogeneous laser profile, resulting in an expectation value of the initial density which is equal at every position, while we still include the random noise related to vacuum fluctuations.

\section{A. Bogoliubov approximation}

As long as the condensate fraction is large, the dynamics can be treated in the linearized Bogoliubov approximation. The field $\phi(x, t)$ is decomposed in a condensate and fluctuations, $\phi(x, t)=\phi_{c}(t)+\delta \phi(x, t)$. The evolution of the condensate density is determined by the equation for the condensate field,

$$
i \hbar d \phi_{c}(t)=\left[-i \frac{\gamma}{2}+g\left|\phi_{c}(t)\right|^{2}\right] \phi_{c}(t) d t,
$$

which yields

$$
\left|\phi_{c}(t)\right|^{2} \equiv n_{c}(t)=n_{c}(0) \exp (-\gamma t \hbar) .
$$

The fluctuations are expanded in Fourier space as

$$
\delta \phi(x, t)=\exp \left[-\frac{i g}{\hbar} \int_{0}^{t} d s n_{c}(s)\right] \frac{1}{\sqrt{L}} \sum_{k} \phi(k, t) e^{i k x},
$$

where $L$ is the length of the one-dimensional wire considered here. The linearized equations of motion for the fluctuations can be written compactly in terms of the vector $\Phi(k)=[\phi(k), \phi(-k)]^{T}$ and the noise vector $d \Xi(k)=$ $[d W(k), d W(-k)]^{T}$ :

$$
i \hbar d \Phi(k)=B(k, t) \Phi(k) d t+\frac{\sqrt{\hbar \gamma}}{2} d \Xi(k),
$$

where the Bogoliubov matrix equals

$$
B(k, t)=\left(\begin{array}{cc}
\varepsilon(k)+g n_{c}(t)-\frac{i \gamma}{2} & g n_{c}(t) \\
-g n_{c}(t) & -\varepsilon(k)-g n_{c}(t)-\frac{i \gamma}{2}
\end{array}\right)
$$

and $\varepsilon(k)=\hbar^{2} k^{2} /(2 m)$. From the solution of these stochastic differential equations, we can compute the time evolution of the correlation functions.

\section{B. Momentum distribution and first-order coherence}

The momentum distribution of the fluctuations is

$$
n(k, t)=\left\langle\psi^{\dagger}(k, t) \psi(k, t)\right\rangle=\left\langle\phi^{*}(k, t) \psi(k, t)\right\rangle-1 / 2 .
$$

For the homogeneous system, the stochastic fields are computed by solving the differential equation (9). In the limit $k \rightarrow 0$ it can be solved exactly, which gives for the momentum distribution,

$$
\lim _{k \rightarrow 0} n(k, t)=2\left(\frac{g n_{c}(0)}{\gamma}\right)^{2} e^{-2 \gamma t / \hbar}\left(e^{\gamma t / \hbar}-\frac{\gamma t}{\hbar}-1\right) .
$$

For large momenta, we resort to the sudden approximation [18], which is applied to a system without losses, but in every other aspect is the same as the polariton system of interest. It is valid when the quench occurs much faster than the other dynamics of the system. Before the quench, the elementary excitations are the polaritons. After the quench, however, Bogoliubov excitations are the elementary excitations of the system. Their evolution can be calculated straightforwardly. An inverse Bogoliubov transformation is then used to find the polariton expectation values at later times. Finally, we have multiplied the result for a lossless system with an exponential decay. This yields a good description of the average value of the momentum distribution,

$$
\left\langle\psi^{\dagger}(k, t) \psi(k, t)\right\rangle=\left[\frac{\left.g n_{c}(0)\right]}{\hbar \omega_{B}(k)}\right]^{2} \sin \left[\omega_{B}(k) t\right]^{2} e^{-\gamma t / \hbar},
$$


where $\hbar \omega_{B}(k)=\sqrt{\varepsilon(k)\left[\varepsilon(k)+2 g n_{c}(0)\right]}$ is the Bogoliubov dispersion. In the next section, we will calculate the secondorder coherence from the momentum distribution and the anomalous average $\langle\psi(k, t) \psi(-k, t)\rangle$. The latter quantity is calculated following the same procedure as for the momentum distribution. Thus we first determine $\langle\psi(k, t) \psi(-k, t)\rangle$ for a system without decay, i.e., $\gamma=0$, and reintroduce the time dependence by letting the expectation values decay exponentially, which yields

$$
\begin{aligned}
\langle\psi(k, t) \psi(-k, t)\rangle= & \frac{g n_{c}(0)}{2\left[\hbar \omega_{B}(k)\right]^{2}} e^{-\gamma t / \hbar}\left\{\hbar \omega_{B}(k) \cos \left[\omega_{B}(k) t\right]^{2}\right. \\
& \left.-\left[\hbar \omega_{B}(k)+\varepsilon(k)+g n_{c}(0)\right] \sin \left[\omega_{B}(k) t\right]^{2}\right\} .
\end{aligned}
$$

The Fourier transform of the momentum distribution gives us the first-order correlation in real space,

$$
g^{(1)}\left(x, x^{\prime}\right)=\frac{\left\langle\psi^{\dagger}(x, t) \psi\left(x^{\prime}, t\right)\right\rangle}{\sqrt{\left\langle\psi^{\dagger}(x, t) \psi(x, t)\right\rangle\left\langle\psi^{\dagger}\left(x^{\prime}, t\right) \psi\left(x^{\prime}, t\right)\right\rangle}},
$$

in terms of the quantum fields. In terms of the stochastic fields, the first-order coherence reads

$$
\begin{aligned}
g^{(1)}\left(x, x^{\prime}\right)= & {\left[\left\langle\phi^{*}(x, t) \phi\left(x^{\prime}, t\right)\right\rangle-\delta_{x, x^{\prime}} /(2 \Delta V)\right] } \\
& \times\left[\left\langle\phi^{\dagger}(x, t) \phi(x, t)-1 /(2 \Delta V)\right\rangle\right. \\
& \left.\times\left\langle\phi^{\dagger}\left(x^{\prime}, t\right) \phi\left(x^{\prime}, t\right)-1 /(2 \Delta V)\right\rangle\right]^{-1 / 2} .
\end{aligned}
$$

At large distances $x-x^{\prime}$, the value of $1-g^{(1)}\left(x, x^{\prime}\right)$ is equal to the quantum depletion, which can also be calculated by integrating over the momentum distribution. The momentum distribution starts out at an approximately constant value, and for momenta larger than the crossover momentum,

$$
k_{*}(t)=\frac{\gamma}{2 \hbar} \sqrt{\frac{m}{g n_{c}(0)}}\left[1-e^{-\gamma t / \hbar}\left(\frac{\gamma t}{\hbar}+1\right)\right]^{-1 / 2},
$$

it can be described by the time average of Eq. (13). Integrating over this two regions, we find [5] for the quantum depletion $\delta n / n_{c}$,

$$
\frac{\delta n(t)}{n_{c}(t)}=C \frac{g^{2} n_{c}(0)}{\gamma^{2}}\left[1-e^{-\gamma t / \hbar}\left(\frac{\gamma t}{\hbar}+1\right)\right] k_{*}(t),
$$

where the constant $C$ is determined numerically. The smallest distance at which the first-order coherence reaches its lowest value is called the coherence length $\ell_{c}$, which is inversely proportional to the crossover momentum,

$$
\ell_{c}(t)=2.1 / k_{*}(t)
$$

where the factor 2.1 was determined numerically.

\section{Second-order coherence in momentum space}

Since the particles are predicted to be produced in pairs with opposite momentum, we expect to find a correlation between polaritons with momentum $k$ and those with momentum $-k$. Therefore we study the second-order coherence in momentum space:

$$
g^{(2)}(k,-k, t)=\frac{\left\langle\psi^{\dagger}(k, t) \psi^{\dagger}(-k, t) \psi(-k, t) \psi(k, t)\right\rangle}{\left\langle\psi^{\dagger}(k, t) \psi(k, t)\right\rangle\left\langle\psi^{\dagger}(-k, t) \psi(-k, t)\right\rangle} .
$$

By applying Wick contraction, we can write the denominator as a product of quadratic expectation values. The nonzero terms are those containing the momentum distribution $\left\langle\psi^{\dagger}(k, t) \psi(k, t)\right\rangle$ and the anomalous average $\left\langle\psi^{\dagger}(k, t) \psi^{\dagger}(-k, t)\right\rangle$. In terms of the stochastic fields, the expression becomes

$$
\begin{aligned}
\left\langle\psi^{\dagger}(k, t) \psi^{\dagger}(-k, t) \psi(-k, t) \psi(k, t)\right\rangle= & \left\langle\phi^{*}(k, t) \phi(k, t)\right\rangle\left\langle\phi^{*}(-k, t) \phi(-k, t)\right\rangle+\left\langle\phi^{*}(k, t) \phi^{*}(-k, t)\right\rangle\langle\phi(-k, t) \phi(k, t)\rangle \\
& -\frac{1}{2}\left\langle\phi^{*}(k, t) \phi(k, t)\right\rangle-\frac{1}{2}\left\langle\phi^{*}(-k, t) \phi(-k, t)\right\rangle+\frac{1}{4} .
\end{aligned}
$$

In the limit $k \rightarrow 0$, an exact solution of Eq. (9) can be found, which yields for the second-order coherence,

$$
\lim _{k \rightarrow 0} g^{(2)}(k,-k, t)=2+\frac{(\gamma t / \hbar)^{2} \exp (-2 \gamma t / \hbar)}{4\left(g n_{c}(0) / \gamma\right)^{2}\left[(1+\gamma t / \hbar)^{2} \exp (-4 \gamma t / \hbar)-2(1+\gamma t / \hbar) \exp (-3 \gamma t / \hbar)+\exp (-2 \gamma t / \hbar)\right]} .
$$

The fraction in this expression diverges both at short times $(t \ll \hbar / \gamma)$, when $\lim _{k \rightarrow 0} g^{(2)}(k,-k, t) \approx 2+\left(g n_{c}(0) t / \hbar\right)^{-2}$, and for long times $(t \gg \hbar / \gamma)$, when $\lim _{k \rightarrow 0} g^{(2)}(k,-k, t) \approx$ $2+\left[\gamma^{2} t / \hbar g n_{c}(0)\right]^{2}$. However, for good cavities with $\gamma \ll$ $g n_{c}(0)$, there is a large time window when $\lim _{k \rightarrow 0} g^{(2)}(k$, $-k, t) \approx 2$.

For large momenta, an expression for the second-order coherence can be calculated from the Wick contracted version of (20) and the results obtained with the sudden approximation (13) and (14). Combining these solutions gives

$$
g^{(2)}(k,-k, t)=2+\left[\frac{\hbar \omega_{B}(k)}{\left.g n_{c}(0)\right]}\right]^{2} \frac{1}{\sin \left[\omega_{B}(k) t\right]^{2}},
$$

and replacing the squared sine function by its average value of one-half yields the following expression for the second-order coherence:

$$
g^{(2)}(k,-k, t)=2+2\left[\frac{\hbar \omega_{B}(k)}{\left.g n_{c}(0)\right]}\right]^{2} .
$$

Here we recognize the inverse of the momentum distribution without the exponential decay. This analytical result can be well understood from the assumption that the particles are indeed produced in pairs. In this case, a polariton with momentum $k$ will always be accompanied by a polariton with momentum $-k$. For large momenta, we expect very few particles. When the expected value is much smaller than 1 , 
only in few of the realizations will a polariton be present, and the cases with more than one polariton with the same momentum will be negligible. Therefore we find that when the polaritons are produced in pairs with opposite momentum, when $\psi^{\dagger}(k, t) \psi(k, t) \ll 1$, and neglecting the exponential decay,

$$
\left\langle\psi^{\dagger}(k, t) \psi^{\dagger}(-k, t) \psi(-k, t) \psi(k, t)\right\rangle \approx\left\langle\psi^{\dagger}(k, t) \psi(k, t)\right\rangle .
$$

As a result, the second-order coherence is the inverse of the momentum distribution,

$$
\left.g^{(2)}(k,-k, t)\right|_{\text {large } k}=\frac{1}{\left\langle\psi^{\dagger}(k, t) \psi(k, t)\right\rangle},
$$

in good agreement with (23). It is interesting to note that the large $k$ limit of the nonequilibrium $g^{(2)}$ from Eq. (23) has the same behavior as in the zero-temperature equilibrium case.

For the homogeneous system, the approximations on top of the Bogoliubov approximation have been checked against numerical calculations that were performed using the Green's function. In terms of the Green's function,

$$
G_{k}\left(t, t^{\prime}\right)=\prod_{j=1}^{N} \exp \left[-i \Delta t B_{k}\left(t_{j}\right) / \hbar\right],
$$

the second-order coherence can be written

$$
\begin{aligned}
g^{(2)}(k,-k, t)= & 1+\frac{1}{4} \mid\left[G_{k}^{\dagger}(t, 0)\right]_{1,2}\left[G_{k}(t, 0)\right]_{1,1}+\left[G_{k}^{\dagger}(t, 0)\right]_{2,2} \\
& \times\left[G_{k}(t, 0)\right]_{1,2}+\frac{\gamma}{\hbar} \int_{0}^{t} d s\left\{\left[G_{k}^{\dagger}(t, s)\right]_{1,2}\right. \\
& \left.\times\left[G_{k}(t, s)\right]_{1,1}+\left[G_{k}^{\dagger}(t, s)\right]_{2,2}\left[G_{k}(t, s)\right]_{1,2}\right\}\left.\right|^{2} \\
& \times\left[\left\langle\psi^{\dagger}(k, t) \psi(k, t)\right\rangle\left\langle\psi^{\dagger}(-k, t) \psi(-k, t)\right\rangle\right]^{-1},
\end{aligned}
$$

where the $x, y$ of $\left[G_{k}(t, s)\right]_{x, y}$ indicate the matrix component. This result is depicted in Fig. 1, together with the analytical expression Eq. (23) derived from the sudden approximation. It can be seen that the analytical expression describes the overall behavior of $g^{(2)}(k,-k, t)$ very well. The maxima of

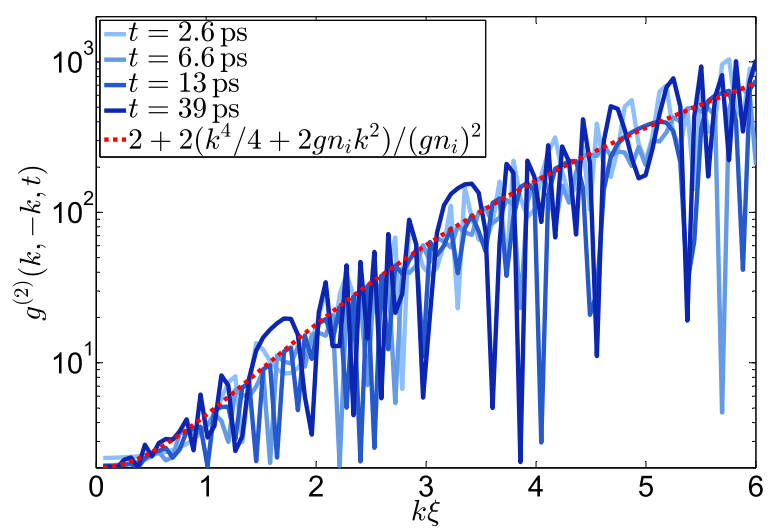

FIG. 1. (Color online) Second-order coherence in momentum space for a homogeneous system calculated with the Green's function method. Solid lines show the numerical results, whereas the dotted line represents Eq. (23). Here, $\xi=\hbar / \sqrt{m g n_{c}(0)}$, and we have chosen $g=0.01 \mu \mathrm{mmeV}, \gamma=0.05 \mathrm{meV}, \hbar=1, m=1, g n_{c}(0) / \gamma=10$. the oscillations are described by two times this expression. From the analytic expression, it can be seen that the period of the oscillations depends both of the time and the momentum. Therefore all osculations at large momenta are fast, whereas the increase of the period as time progresses is more clearly visible for small momenta. These oscillations are expected to be more averaged in experimental data, which would then become closer to the analytically calculated average.

\section{INHOMOGENEOUS SYSTEM}

\section{A. Monte Carlo simulation}

Although the Green's function method provides a good description of the homogeneous system, it has some limitations. First, the interaction energy should not be too high, since the Bogoliubov approximation is no longer valid when the quantum depletion becomes too large. Secondly, the Green's function method becomes cumbersome for inhomogeneous systems. In order to overcome these problems, we have also implemented a Monte Carlo simulation algorithm [19].

In the truncated Wigner-Monte Carlo algorithm, the expectation values are calculated by averaging over many realizations of the system. For the initial situation, an average density is chosen and random noise is added to account for the stochastic nature of the fields. As opposed to the Green's function method, where Eq. (9) was solved, the Monte Carlo algorithm uses both the real- and momentum-space representation of the stochastic fields. The evolution due to interactions and decay, which are the time-dependent parts of the Hamiltonian, is calculated in real space, whereas the effect of the kinetic term is calculated in momentum space. This method has the advantage that we do not have to distinguish between condensate and excitations in the interaction term. In order for the algorithm to work, the time steps for which the evolution is calculated should be small, in order for the effect of sequentially calculating the evolution in real and momentum space to be small. For the homogeneous system we have used a system length of $200 \mu \mathrm{m}$ and $0.4 \mu \mathrm{m}$ as the size of a unit cell. For the inhomogeneous systems, we adapted the length and grid size in order to have sufficient detail, the boundaries of the system distant enough with respect to the width of the Gaussian distribution, while keeping the number of grid points equal to 256 for computational efficiency.

First we verified the results of the Green's function method for the homogeneous system (see Fig. 2). For small momenta, the two methods show a good agreement for all times. However, for larger momenta, the Monte Carlo method predicts more particles than the Green's function method. Nevertheless, both methods still predict few particles with large momenta. The reason why the difference is larger for large momenta and late times is that when few particles are present, the noise becomes relatively more important. Since for the (very) large momenta, the results of the Monte Carlo simulations are mainly due to the noise, this provides a good indication of the overall error in the momentum distribution. Furthermore, in the homogeneous case, we have seen that the difference between the latest times is very small, $t=105$ and $39 \mathrm{ps}$ [5]. Therefore we have chosen to calculate up to $t=39$ ps with the Monte Carlo method. 


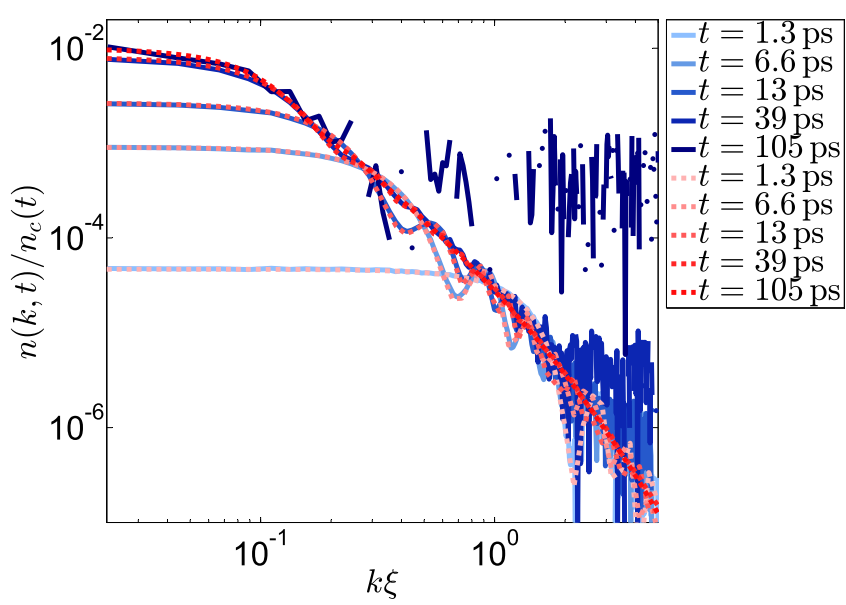

FIG. 2. (Color online) Momentum distribution for a homogeneous system calculated with the Greens' function method (red dotted) and Monte Carlo simulations (blue solid), averaged over 40000 realizations. Here, $\xi=\hbar / \sqrt{m g n_{c}(0)}$, and we have chosen $g=0.01 \mu \mathrm{mmeV}, \gamma=0.05 \mathrm{meV}, L=200 \mu \mathrm{m}, \hbar=$ $1, m=1, g n_{c}(0) / \gamma=10$.

\section{B. Numerical results}

In addition to the homogeneous systems, Monte Carlo simulations were applied to study systems with an initial Gaussian density distribution, given by $n_{i} \exp \left(-x^{2} / s^{2}\right)$, where the time evolution was determined by Eq. (5). The results presented here are obtained from 10000 realizations. The values $s=100$ and $20 \mu \mathrm{m}$ have been chosen for the width of the distribution, and $n_{i}=50 \mu \mathrm{m}^{-1}$ for the initial central density. With this initial density, the Bogoliubov approximation and truncated Wigner approximation were still valid for the homogeneous system. For $s=100 \mu \mathrm{m}$, the behavior in real space can be well understood. The density at the center of the Gaussian decreases faster than the overall exponential decay, whereas the density at the sides of the distributions shows a small relative increase (see Fig. 3). This would be expected from the repulsive interactions. As a result, the density distribution becomes more homogeneous. In the case of a smaller Gaussian

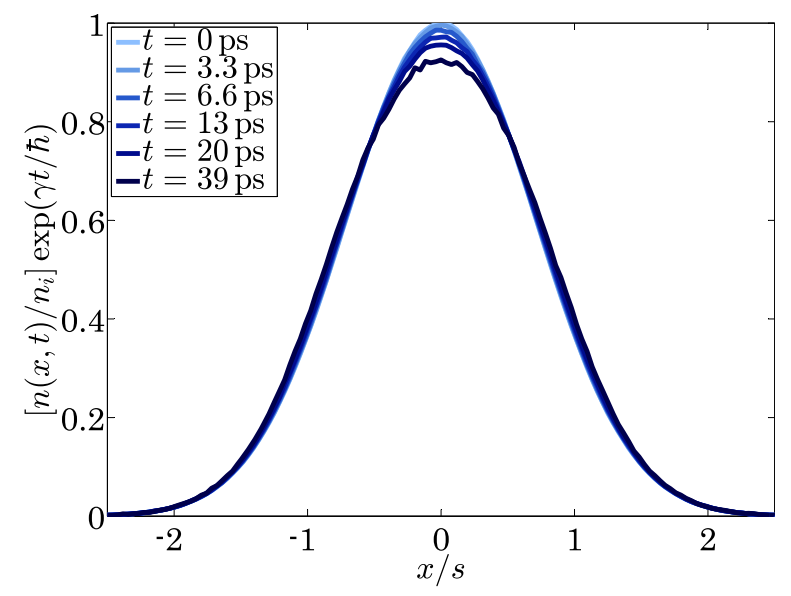

FIG. 3. (Color online) Density calculated with Monte Carlo simulations, initial Gaussian profile given by $n_{i} \exp \left(-x^{2} / s^{2}\right)$, with $s=$ $100 \mu \mathrm{m}$. We have chosen $g=0.01 \mu \mathrm{m} \mathrm{meV}, \gamma=0.05 \mathrm{meV}, \hbar=$ $1, m=1, g n_{i} / \gamma=10$.

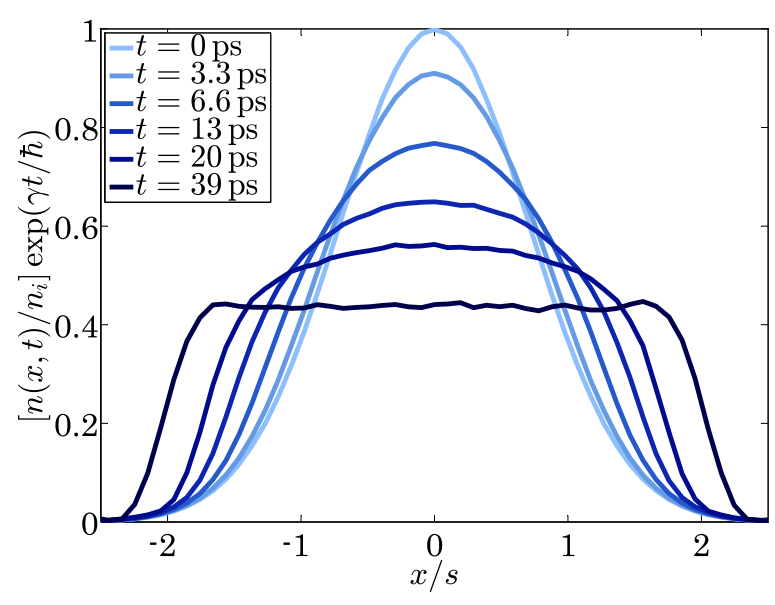

FIG. 4. (Color online) Density calculated with Monte Carlo simulations, initial Gaussian profile given by $n_{i} \exp \left(-x^{2} / s^{2}\right)$, with $s=$ $20 \mu \mathrm{m}$. We have chosen $g=0.01 \mu \mathrm{mmeV}, \gamma=0.05 \mathrm{meV}, \hbar=$ $1, m=1, g n_{i} / \gamma=10$.

distribution, where $s=20 \mu \mathrm{m}$, this effect is even stronger (see Fig. 4). At the latest depicted time, $t=39 \mathrm{ps,} \mathrm{the} \mathrm{central}$ region is very homogeneous and shows a fast decrease at the edges of the distribution. Since the background is completely homogeneous, the oscillations on top of the smooth density curves are related to the errors due to the noise that has not completely been averaged out.

The first-order coherence for the $s=100 \mu \mathrm{m}$ system can be described by the results obtained from the homogeneous system [5]. At short distances, the shape of the first-order coherence is similar for the homogeneous and the Gaussian system. However, at larger distances, the first-order coherence goes back to 1 for the inhomogeneous system, whereas for the homogeneous system it remained constant. Since the two systems are similar at short distances, we still use the term coherence length to refer to the distance at which the first-order coherence is minimal and use the depletion as 1 minus this minimal value. Using the expressions from the homogeneous case with the density at the maximum of the distribution $n_{i}$, we see that the coherence length and the depletion of these analytical expressions are close to the numerical results. Furthermore, there is still a linear relation between the coherence length and the depletion. Therefore a simple correction to the numerical constants would give an even better description of these quantities. The overall shape of $g^{(1)}(x,-x, t)$ is a direct result from the Gaussian shape of the density. For the homogeneous system, a formula for the depletion at very large times as a function of the blueshift $g n_{c}(t)$ was derived [see Eq. (18)]:

$$
\lim _{t \rightarrow \infty} \delta n(t) / n_{c}(t) \approx 0.77 \mathrm{~g} /(\xi \gamma) .
$$

In the inhomogeneous system the healing length $\xi=$ $\hbar / \sqrt{m g n_{c}(0)}$ becomes position dependent. Consequently, the final depletion will also depend on the position. When $n_{c}(t=$ 0 ) is simply replaced by the initial Gaussian distribution, $n_{i} \exp \left(-x^{2} / s^{2}\right)$, the black dashed lines from Figs. 5 and 6 are found. For the wider Gaussian, with $s=100 \mu \mathrm{m}$, this describes the behavior of the first-order coherence very well at late times. Therefore the fact that the coherence returns to 


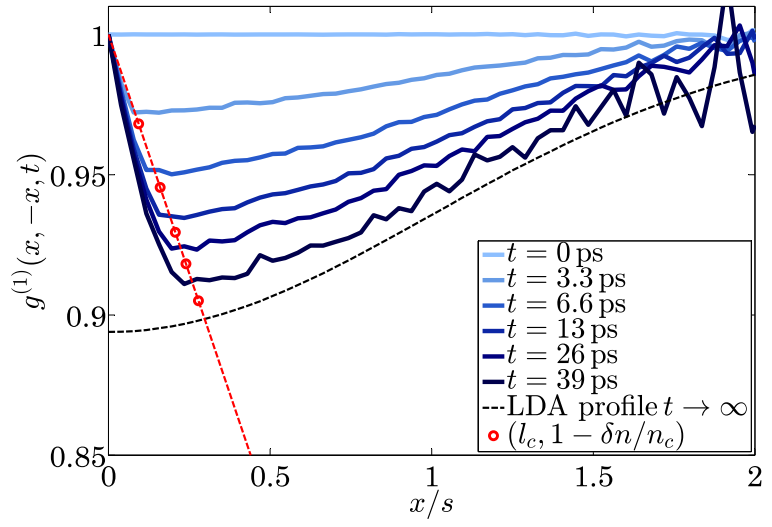

FIG. 5. (Color online) First-order spatial coherence $g^{(1)}(x,-x, t)$ for an initial Gaussian distribution $\left[n_{i} \exp \left(-x^{2} / s^{2}\right)\right]$ with width $s=$ $100 \mu \mathrm{m}$, calculated with Monte Carlo simulations. The (red) dots correspond to $\ell_{c}(t)$ as given in Eq. (19), and $\delta n(t) / n_{i}(t)$ in Eq. (18) at the same times as the (blue) solid lines, where $n_{i}$ was used instead of $n_{c}(0)$. The (black) dashed lines depicts $g^{(1)}(x,-x, t \rightarrow \infty)$, based on Eq. (29), with $n_{i} \exp \left(-x^{2} / s^{2}\right)$ instead of $n_{c}(0)$. Same parameters as Fig. 3.

1 for distances comparable to the width of the distribution is due to the small density, which leads to a smaller depletion.

However, for the smaller Gaussian, where $s=20 \mu \mathrm{m}$, the local density approximation is no longer valid. Nevertheless, the initial decay of the first-order coherence, until $x \approx$ $20 \mu \mathrm{m}$, is still described by the linear relation found in the homogeneous case.

In momentum space, on the other hand, the system with a Gaussian density is very different from a homogeneous system. This is in accordance with expectations, since for an initial Gaussian distribution, many momentum states are occupied from the start. Moreover, the inhomogeneous density profile leads to an expulsion of the polaritons away from the region where they were created. This acceleration corresponds to a shift of the momentum distribution. Hence, momentum

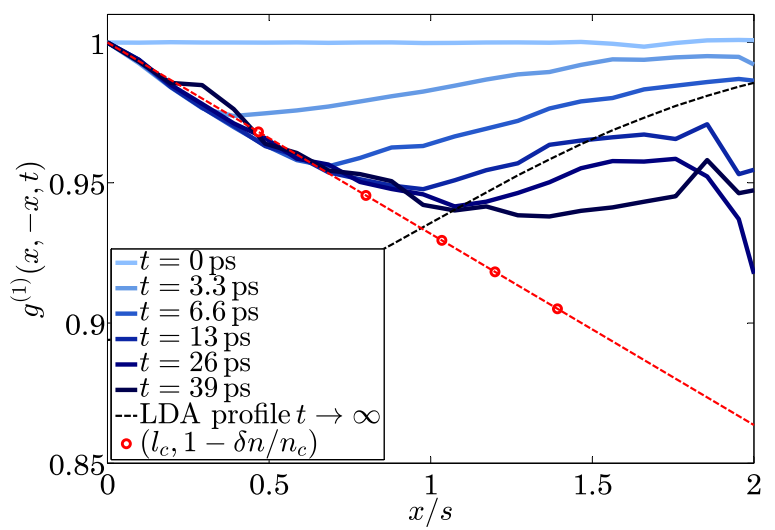

FIG. 6. (Color online) First-order spatial coherence $g^{(1)}(x,-x, t)$ for an initial Gaussian distribution $\left[n_{i} \exp \left(-x^{2} / s^{2}\right)\right]$ with width $s=$ $20 \mu \mathrm{m}$, calculated with Monte Carlo simulations. $\ell_{c}(t)$ is given in Eq. (19), and $\delta n(t) / n_{i}(t)$ in Eq. (18), where $n_{i}$ was used instead of $n_{c}(0)$ and the values for $t$ are the same as for the (blue) solid lines. The (black) dashed lines depicts $g^{(1)}(x,-x, t \rightarrow \infty)$, based on Eq. (29) with $n_{i} \exp \left(-x^{2} / s^{2}\right)$ instead of $n_{c}(0)$. Same parameters as Fig. 4.

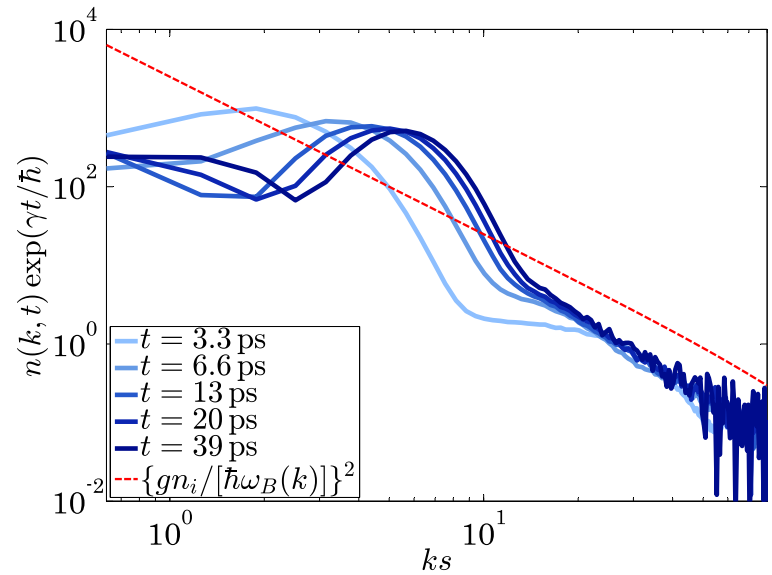

FIG. 7. (Color online) Momentum distribution for an initial Gaussian distribution $\left[n_{i} \exp \left(-x^{2} / s^{2}\right)\right]$ with width $s=100 \mu \mathrm{m}$, calculated with Monte Carlo simulations. Same parameters as Fig. 3.

conservation in the interactions no longer results in pair production of polaritons with opposite momentum. In Figs. 7 and 8 we see an increase of the particle number with respect to the homogeneous case at small yet finite momentum, which indicates that the distribution is expanding, which is indeed what was seen in the evolution of the density. The wider and the smaller distribution both display very similar behavior, where the smaller density distribution has a peak in the momentum distribution at larger momenta, as compared to the wider density distribution. At larger momenta, the momentum distribution follows that of the homogeneous system more closely for the $s=100 \mu \mathrm{m}$ case. However, the particle number at these momenta is very small.

The second-order coherence, depicted in Figs. 9 and 10, is even more different from the homogeneous case. In these figures, the (red) dotted line represents the analytically calculated $g^{(2)}(k,-k, t)-1$ from Eq. (23) for the homogeneous system. One is subtracted, since for small momenta, the numerically calculated second-order coherence is close to 1 , the value that corresponds to a coherent system. We see that the second-order coherence remains close to this value, even for momenta at which the momentum distribution seems to resemble the

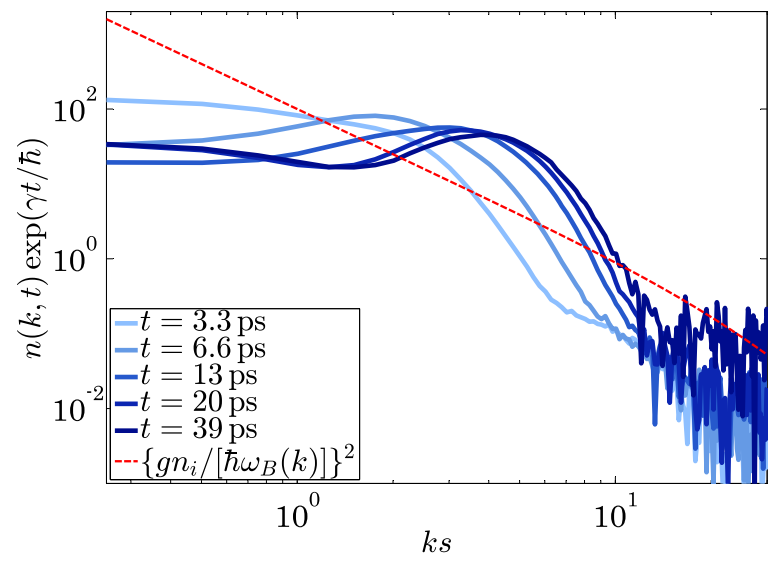

FIG. 8. (Color online) Momentum distribution for an initial Gaussian distribution $\left[n_{i} \exp \left(-x^{2} / s^{2}\right)\right]$ with width $s=20 \mu \mathrm{m}$, calculated with Monte Carlo simulations. Same parameters as Fig. 4. 


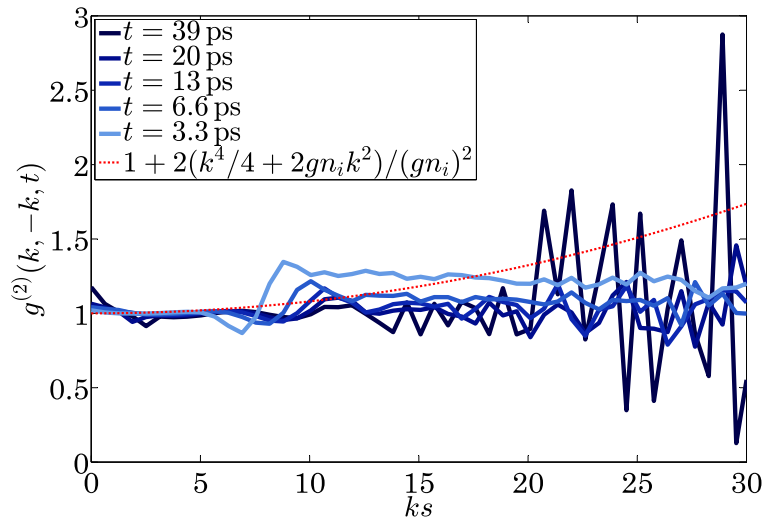

FIG. 9. (Color online) Second-order coherence in momentum space for initial Gaussian distribution $\left[\exp \left(-x^{2} / s^{2}\right)\right]$ with width $s=$ $100 \mu \mathrm{m}$, calculated with Monte Carlo simulations. Same parameters as Fig. 3.

homogeneous case for the wider density distribution. This suggests that even for these momenta, most particles are part of the condensate. For the system with $s=20 \mu \mathrm{m}$, the second-order coherence, is either close to 1 or it contains too much noise for a good description.

\section{CONCLUSIONS}

We have studied a quantum quench consisting of a sudden injection of polaritons in a microcavity. Both a homogeneous and a Gaussian initial density distribution have been examined. The homogeneous case has been related to the dynamical Casimir effect previously. Where the correlation functions in the homogeneous case could still be calculated analytically, we performed truncated Wigner-Monte Carlo simulations for the Gaussian excitation pulse. The first-order spatial coherence

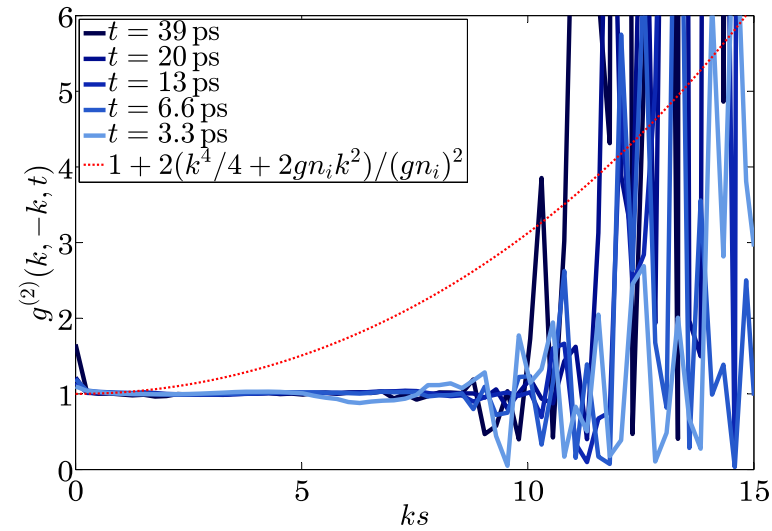

FIG. 10. (Color online) Second-order coherence in momentum space for initial Gaussian distribution $\left[\exp \left(-x^{2} / s^{2}\right)\right]$ with width $s=$ $20 \mu \mathrm{m}$, calculated with Monte Carlo simulations. Same parameters as Fig. 4.

is well approximated by the local density approximation for a sufficiently wide pulse. The second-order coherence in momentum space evidences the production of excitations in pairs in the homogeneous system. For a system with an initial Gaussian density distribution, multiple momentum states are significantly occupied from the start. Here, the second-order coherence indicates that many of these particles are coherent, so that evidence of quantum correlations is highly suppressed.

\section{ACKNOWLEDGMENTS}

The authors thank I. Carusotto, M. Van Regemortel, and D. Sels for stimulating discussions and acknowledge financial support from the FWO Odysseus program.
[1] A. Polkovnikov, K. Sengupta, A. Silva, and M. Vengalattore, Rev. Mod. Phys. 83, 863 (2011).

[2] T. Langen, R. Geiger, and J. Schmiedmayer, Annu. Rev. Condens. Matter Phys. 6, 201 (2015).

[3] I. Bloch, J. Dalibard, and W. Zwerger, Rev. Mod. Phys. 80, 885 (2008).

[4] I. Carusotto and C. Ciuti, Rev. Mod. Phys. 85, 299 (2013).

[5] S. Koghee and M. Wouters, Phys. Rev. Lett. 112, 036406 (2014).

[6] G. Moore, J. Math. Phys. 11, 2679 (1970).

[7] V. V. Dodonov, Phys. Scr. 82, 038105 (2010).

[8] C. M. Wilson, G. Johansson, A. Pourkabirian, J. R. Johansson, T. Duty, F. Nori, and P. Delsing, Nature (London) 479, 376 (2011).

[9] H. S. Nguyen, D. Gerace, I. Carusotto, D. Sanvitto, E. Galopin, A. Lemaître, I. Sagnes, J. Bloch, and A. Amo, Phys. Rev. Lett. 114, 036402 (2015).
[10] W. G. Unruh, Phys. Rev. Lett. 46, 1351 (1981).

[11] J. Steinhauer, Nat. Phys. 10, 864 (2014).

[12] C.-L. Hung, V. Gurarie, and C. Chin, Science 341, 1213 (2013).

[13] A. Sakharov, JETP 49, 345 (1965).

[14] T. Langen, R. Geiger, M. Kuhnert, B. Rauer, and J. Schmiedmayer, Nat. Phys. 9, 640 (2013).

[15] A. Sinatra, C. Lobo, and Y. Castin, J. Phys. B 35, 3599 (2002).

[16] A. Polkovnikov, Ann. Phys. (NY) 325, 1790 (2010).

[17] P. B. Blakie, A. S. Bradley, M. J. Davis, R. J. Ballagh, and C. W. Gardiner, Adv. Phys. 57, 363 (2008).

[18] I. Carusotto, R. Balbinot, A. Fabbri, and A. Recati, Eur. Phys. J. D 56, 391 (2010).

[19] I. Carusotto and C. Ciuti, Phys. Rev. B 72, 125335 (2005). 